\title{
Stage 0 Testicular Cancer AJCC v6 and v7
}

National Cancer Institute

\section{Source}

National Cancer Institute. Stage O Testicular Cancer A/CC v6 and v7. NCI Thesaurus.

Code C4523.

Stage 0 includes: PT is, NO, MO, S0. pT is: Intratubular germ cell neoplasia (carcinoma in situ). N0: regional lymph node metastasis. MO: No distant metastasis. S0: Marker study levels within normal limits. (AJCC 6th and 7th eds.) 